Correction

\title{
Correction: Interaction between physical activity, PITX1 rs647161 genetic polymorphism and colorectal cancer risk in a Korean population: a case-control study
}

\author{
Madhawa Neranjan Gunathilake ${ }^{1}$, Jeonghee Lee ${ }^{2}$, Young Ae Cho², Jae Hwan Oh${ }^{3}$, \\ Hee Jin Chang ${ }^{3}$, Dae Kyung Sohn ${ }^{3}$, Aesun Shin ${ }^{4}$ and Jeongseon Kim ${ }^{2}$ \\ ${ }^{1}$ Department of Cancer Control and Population Health, Graduate School of Cancer Science and Policy, Goyang-si, Gyeonggi- \\ do 10408, South Korea \\ 2 Department of Biomedical Science, Graduate School of Cancer Science and Policy, Goyang-si, Gyeonggi-do 10408, South \\ Korea \\ ${ }^{3}$ Center for Colorectal Cancer, National Cancer Center Hospital, National Cancer Center, Goyang-si, Gyeonggi-do 10408, \\ South Korea \\ ${ }^{4}$ Department of Preventive Medicine, Seoul National University College of Medicine, Jongno-gu, Seoul 03080, South Korea \\ Published: October 09, 2018
}

Copyright: Gunathilake et al. This is an open-access article distributed under the terms of the Creative Commons Attribution License 3.0 (CC BY 3.0), which permits unrestricted use, distribution, and reproduction in any medium, provided the original author and source are credited.

This article has been corrected: The correct Funding information is given below:

\section{FUNDING}

This work was supported by grants from the National Cancer Center, Korea (1630960, 1710882, and 1810090), the National Research Foundation of Korea (2015R1A5A6001906, 2010-0010276, and 2013R1A1A2A10008260), and the fund from the Ministry of Food and Drug Safety (17162MFDS040).

Original article: Oncotarget. 2018; 9:7590-7603. https://doi.org/10.18632/oncotarget.24136 